\title{
Article \\ Optimization of Tomato Productivity Using Flowering Time Variants
}

\author{
Sujeevan Rajendran ${ }^{1,+}$, Jung Heo ${ }^{1,+}$, Yong Jun Kim ${ }^{1}$, Dae Heon Kim ${ }^{2} \mathbb{D}$, Kisung Ko ${ }^{3}$, Young Koung Lee ${ }^{4} \mathbb{D}$, \\ Seok Kwi Oh ${ }^{5}$, Chul Min Kim ${ }^{5}$ (D), Jong Hyang Bae ${ }^{5}$ and Soon Ju Park ${ }^{1, *}$
}

1 Division of Biological Sciences, Wonkwang University, Iksan 54538, Korea; sujeevr@outlook.com (S.R.); dandy3745@naver.com (J.H.); youngjun6381@naver.com (Y.J.K.)

2 Department of biology, Sunchon National University, Sunchon 57922, Korea; dheonkim@scnu.ac.kr

3 Department of Medicine, Therapeutic Protein Engineering Lab, College of Medicine, Chung-Ang University, Seoul 06974, Korea; ksko@cau.ac.kr

4 Institute of Plasma Technology, Korea Institute of Fusion Energy, 37, Dongjangsan-ro, Gunsan-si 54004, Korea leeyk@nfri.re.kr

5 Division of Horticulture Industry, Wonkwang University, Iksan 54538, Korea; ohsh6412@wku.ac.kr (S.K.O.); chulmin21@wku.ac.kr (C.M.K.); bae@wku.ac.kr (J.H.B.)

* Correspondence: sjpark75@wku.ac.kr

+ These authors contributed equally to this work.

check for

updates

Citation: Rajendran, S.; Heo, J.; Kim, Y.J.; Kim, D.H.; Ko, K.; Lee, Y.K.; Oh, S.K.; Kim, C.M.; Bae, J.H.; Park, S.J. Optimization of Tomato Productivity Using Flowering Time Variants. Agronomy 2021, 11, 285. https:// doi.org/10.3390/agronomy11020285

Received: 26 September 2020

Accepted: 1 February 2021

Published: 4 February 2021

Publisher's Note: MDPI stays neutral with regard to jurisdictional claims in published maps and institutional affiliations.

Copyright: (c) 2021 by the authors. Licensee MDPI, Basel, Switzerland. This article is an open access article distributed under the terms and conditions of the Creative Commons Attribution (CC BY) license (https:// creativecommons.org/licenses/by/ $4.0 /)$.

\begin{abstract}
The control of flowering time is a major contributing factor to the improvement of crop yield by optimizing plant growth in a crop cycle. Genetic variants that determine flowering time can provide insights into optimizing flowering time for higher yields and other beneficial traits in tomato crops. Here, we examined a collection of flowering time variants to assess their effects on biomass and total tomato yields. Five late flowering (lf), thirteen large plant (lp), and seven floral homeotic $(f h)$ mutants were identified as flowering time variants that could be rearranged according to leaf production in the primary shoot meristem (PSM). A flowering time continuum of mutants was translated into a positive continuum of biomass yield with more leaves, branches, and floral organs. The flowering time continuum showed an optimal curve of fruit yield, indicating a certain late flowering time as optimal for fruit yield, with the yield gradually decreasing in both directions with earlier or later flowering times. We isolated lf1,lf10,lp22, and $f h 13$ as high-yielding genotypes with optimal flowering time, showing a new balance between the vegetative and flowering phases of tomato. Additionally, $l p 8$, fh 8 , and $f h 15$ produced extremely high biomass in leaves, axillary shoots, and floral organs due to late flowering in shoot apices with additional production of floral organs and lateral shoot. Our new late-flowering variants provide new genetic resources that can be used to optimize crop yield by fine-tuning flowering time, and future molecular studies could be conducted by revisiting our yield model.
\end{abstract}

Keywords: flowering time; genetic variants; optimization; plant growth; tomato yield

\section{Introduction}

In flowering crops, the transition to flowering, which is widely known as flowering time, is a major change in determining crop yields by optimizing plant growth in a crop cycle. Plant growth and development can be divided into the vegetative and reproductive phases. Apical and lateral vegetative meristems consecutively produce leaves and branches until vegetative meristems irreversibly transform into reproductive meristems, at which point the meristem initiates the production of inflorescences, flowers, and ultimately fruits. With this system, the reproductive success of flowering plants depends on the transition time from the vegetative to the reproductive phase $[1,2]$.

Genetic variants that determine flowering time have provided resources for the optimization of flowering time for higher yields and biomass of crops. Universal late-flowering mutants in the flowering signal pathway, such as flowering locus $t(f t)$ and heading date 
$3 a(h d 3 a)$, normally produce more vegetative and reproductive organs but fewer seed sets because of over-productive vegetative growth [3,4]. However, optimized transitional variants in Grain number, plant height, and heading date 7 (Ghd7) [5,6], Heading date 1 (Hd1) [7], and Days to Heading 7 (DTH7) [8,9], which regulate the florigen pathway, expressed relatively few florigen signals, resulting in minor late-flowering phenotypes with high yield potential in rice (Oryza sativa L.). In several ecotypes of Arabidopsis [Arabidopsis thaliana (L.) Heynh], genetic variations in key genes regulating floral transition, such as vernalization and photoperiod-dependent floral promotion pathways, cause adaptation to seasonal cues in different geographic regions [10]. Cultivated tomatoes (Solanum lycopersicum L.), known as a day-neutral plant, have lost their photoperiodic sensitivity because of the mutation in SELF PRUNING 5G (SP5G) during the domestication of wild tomato species. It has been suggested that tomatoes adapt to the temporal region through a loss of sensitivity to day length [11].

Sympodial plants, such as tomato, transition to inflorescence on the shoot apices multiple times in a lifespan [12]. This produces many vegetative sympodial/axillary shoots with multi-flowered inflorescences for the next generation. Therefore, mutations in flowering time regulators usually lead to significant differences in shoot growth in tomato. A mutant, named self-pruning ( $s p$ ), induced precocious shoot termination with an early floral transition in sympodial shoot meristems (SYMs) and axillary shoot meristems (AXMs), has been used for field tomato breeding because farmers prefer self-pruning and identical fruit maturation for mechanical harvesting [13,14]. SINGLE FLOWER TRUSS (SFT) is tomato florigen, and sft shows the extreme delay of transition time to flowering. The $\mathrm{sft} /+$ tomato plants showed minor delays in the transition time in all sympodial shoots and axillary shoots due to multiple floral transitions. These changes in the whole plant system that induced more vegetative shoots and floral organs resulted in a hybrid vigor yield effect with optimal transition time to flowering [15]. Moreover, the Suppressor of SP (SSP), an ortholog of FLOWERING LOCUS D (FD), forms the tomato Florigen Activation Complex (FAC) with SFT and 14-3-3/54 proteins to induce floral transition [16]. Genetic dosages of florigen activation were generated by crossing $s f t$, ssp, and wild type, which determined the continuum of flowering time and the extent of sympodial shoot growth from precocious termination to indetermination. In addition to SP, three SP5Gs repress florial transition in Nicotiana benthamiana as functional antiflorigen, and the SP5Gs expressions were mediated by $P H Y B$ in tomato [17]. $C R$-sp5g $s p$ additively enhanced faster axillary shoot flowering than $s p$ single mutants, resulting in a compact determinate growth habit in tomato [11]. The relevant dosages of flowering signals could be used to isolate genotypes by determining an optimal yield from yield trends under the optimal floral transition time [1,18].

Recently, tomato mutant populations produced using ethyl methanesulfonate (EMS) mutagenesis or gamma-ray irradiation have been investigated and categorized with visible phenotypes. Since these mutant resources are open resources for molecular and genetic research, flowering time, plant size, and floral homeotic mutants could be used as new genetic resources for future tomato breeding and for cloning mutant genes for functional studies [19-21]. procera 2 (pro 2), a mutant from a Micro-Tom mutant population, was identified as a variant that displays a relatively large plant size and high fruit yield variant under high-temperature conditions, resulting from a new, milder, hypomorphic allele of SIDELLA [22]. ssp, a mutant from another mutant population, was also identified as large in plant size due to late-flowering, and mutations in SSP and ssp/+ sft/+ increased tomato fruit yields with optimal flowering time and growth [1].

The characteristics of Micro-Tom are compact plant size and relatively early flowering, due to three major recessive mutations: $d w a r f(d), s p$ (self pruning), and, putatively, miniature (mnt) [23]. Micro-Tom is a suitable model for studies into correlations between flowering time, plant size, and tomato yield using publicly available mutant resources. Changes in plant size, flowering time, and yield can be clearly identified with visual and/or basic instrumental measurements. 
Based on available studies and mutant materials, we hypothesized that the lateflowering variants would display suppressed shoot terminations, inducing biomass and fruit yield changes in Micro-Tom (Figure 1a). Optimization of yields could be achieved by fine-tuning transition time using flowering time variants. In this study, we rescreened late-flowering variants from potential candidates collected in the TOMATOMA mutant database. The mutants of late floral transition showed associations between the period of vegetative leaf and axillary branch production and an increase in biomass in three categories: late-flowering, large plant, and floral homeotic phenotypes. We demonstrated that incremental later transitions drove a gradual increase in fruit yield until it reached an optimum, after which the yield progressively decreased in late-flowering variants.

a
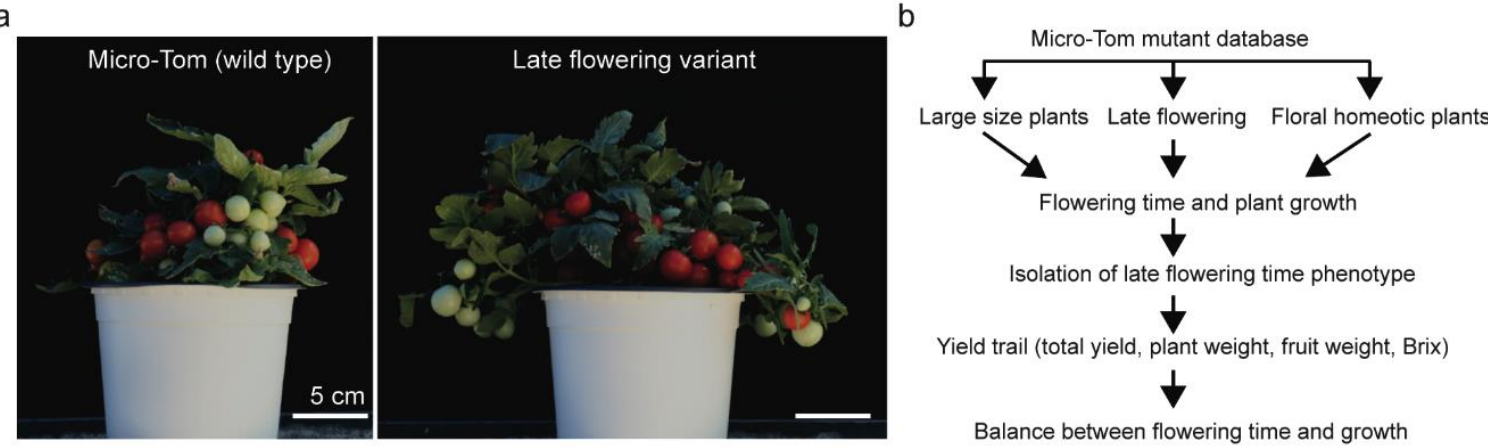

Figure 1. Strategy of fine-tuning optimal tomato yields through regulating flowering time and plant growth in tomato. (a) Representative plants with fruit set in wild type (left; Micro-Tom) and in weakly late-flowering mutant (Right). (b) The workflow followed to elucidate the relationship between plant weight, total yield, and other yield-related traits with flowering time variants.

\section{Materials and Methods}

\subsection{Plant Materials and Growth}

A total of 55 Micro-Tom mutants, induced by EMS and gamma-ray irradiation, were obtained from TOMATOMA (NBRP tomato: http:/ / tomato.nbrp.jp/indexEn.html (accessed on 3 February 2021)). All plants were grown under long-day conditions and controlled temperatures in a greenhouse at Wonkwang University, Iksan, South Korea. Plants were grown under natural light and supplemental light from Natrium and halogen lamps applied in the early morning and late evening. The light/dark cycle was $16 \mathrm{~h} / 8 \mathrm{~h}$ per day. Plants were supplied with nutrients in the irrigation water from one month after transplanting, following the manufacturer's guidelines (S-feed-Hannong; $1 \mathrm{~kg} / 10 \mathrm{a} /$ day; https: / / www.farmhannong.com/kor/product/product_ct01/view.do?seq=4392 (accessed on 3 February 2021)).

\subsection{Phenotyping}

Flowering time, shoot determinacy, and axillary shoot numbers were collected from mature plants at 40 days after transplanting (DAT) into the soil. Flowering time was determined using leaf production on the primary shoot before flowering. To determine plant size, the plant body was measured using an electronic balance after removing the roots and fruits. Floral homeotic lines were classified by the mutant's phenotypic deviation from the wild type floral or inflorescence structure. Deviations were identified and classified according to inflorescence length, inflorescence branches, and floral organ number. Shoot determinacy was identified by the termination of the apical meristem on the main shoot. The number of axillary shoots was counted as the number of axillary shoots produced by the main shoot. The main shoots consisted of primary shoots and successive sympodial shoots that grew out before the shoot termination. All the data were collected from at least three biological replicates, except for a few mutant lines, due to genetic segregation. 
Representative images were captured at $40 \mathrm{DAT}$ and at the time of harvesting using a digital camera (Canon EOS 80D).

\subsection{Quantitative Real-Time RT-PCR}

To extract RNA, mature leaves were collected from 6 weeks old plants after transplanting at $10 \mathrm{am}$ in the greenhouse. Total RNA was extracted using the AccuPrep ${ }^{\circledR}$ Universal RNA extraction kit (Bioneer, Daejeon, Korea) and treated with RNase-free DNase to remove DNA fragments (Qiagen, Hilden, Germany). One microgram of total RNA was used to synthesize cDNA with ReverTra Ace- $\alpha^{\circledR}$ (TOYOBO, Osaka, Japan). Semi-quantitative RT-PCR was conducted using KOD plus polymerase (TOYOBO, Osaka, Japan) to amplify the transcripts. Amplified products were visualized by ethidium bromide staining. Quantitative real-time RT-PCR was conducted using a T100 ${ }^{\mathrm{TM}}$ Thermocycler system (Bio-Rad, Hercules, CA, USA). Primer information is given in Table S1. Reactions ( $10 \mu \mathrm{L}$ final volume) were prepared using $5 \mu \mathrm{L}$ of $\mathrm{iQ}^{\mathrm{TM}} \mathrm{SYBR}^{\circledR}$ Green PCR Master Kit (Applied Biosystems, Foster City, CA, USA), 0.5 pmol of a primer pair, and $1 \mu \mathrm{L}$ of cDNA template. Three biological replicates were used for the quantifications. Ubiquitin was used as the reference. Transcript-specific primer information is given in Table S1.

\subsection{Yield Trials}

Yield trials were conducted at Wonkwang University, Iksan, South Korea. Three yield trials were conducted in a greenhouse, as previously described [24]. The first yield trial was conducted from March to June, and the second yield trial was conducted from August to November. All mutant and wild-type lines were planted in the randomized groups. All mutants were grown in at least six replicates for each experiment. Seedlings were grown in 50-hole seed trays for 24 days and transplanted into pre-prepared land. Plant growth was controlled using standard drip irrigation and fertilizer regimes. Each individual plant was spaced 0.4 square meters apart. Defective and/or diseased seedlings or plants were removed from the experiments. For the third experiment, all plants were grown in pots (height: $14.1 \mathrm{~cm}$, diameter: $13.7 \mathrm{~cm}$ ) filled with approximately $250 \mathrm{~g}$ of potting material (Santo-Heungnung Bio), with a controlled water and nutrient system. All plants were grown as mentioned above under nursery conditions and transplanted into filled pots. Water was performed regularly and uniformly. Nutrients were applied three times a week with a recommended liquid fertilizer mix (19-19-19/S-feed-Hannong industries).

\subsection{Harvesting and Data Collection}

Harvesting was conducted when Micro-Tom (control plants) showed at least $60 \%$ ripening. Plant weight and fruit yield were measured after the roots and fruits were manually removed from the soil and plant. Estimation of the average fruit weight and total soluble sugar content (Brix) in fruit juice was determined by randomly selecting ten fruits. All fruits, including red and green fruits, were weighed to measure the total fruit yield. Another set of ten red fruits was randomly selected to estimate the average fruit weight. The Brix value (\%) was quantified using a digital Brix refractometer (ATAGO Palette).

\subsection{Statistical Analysis}

Statistical calculations were performed using the JMP 14.3.0 software package (SAS Institute, Cary, NC, USA). Student's t-test (two-tailed) was used for comparison with the wild type. The Tukey-Kramer multiple-comparison test was used for multiple comparisons using the Fit $\mathrm{Y}$ by $\mathrm{X}$ function in the JMP package. ANOVA was performed using the fit model function of the JMP 14.3.0 software package. Bivariate correlation analyses were conducted to evaluate associations among all five traits in all seasons. Non-linear regression was used to estimate a model with flowering time as the independent variable and total yield or plant weight as dependent variables. Bivariate correlation analyses and nonlinear regression were run using IBM ${ }^{\circledR}$ SPSS ${ }^{\circledR}$ Statistics 24 (IBM Corp., Armonk, NY, USA. 
Released 2016. IBM SPSS Statistics for Windows, Version 24.0). Population parameters were estimated using linear and quadratic equations with default parameters.

\section{Results}

\subsection{Isolation of Late-Flowering Variants from Micro-Tom Mutants}

We developed an experimental workflow to isolate optimal genotypes via the process of fine-tuning a new balance between vegetative and reproductive production in a quantitative manner, after screening late-flowering mutants using late flowering $(l f)$, large plant $(l p)$, and floral homeotic (fh) mutants (Figure 1b).

To isolate genetic resources of flowering time variants from the publicly available genetic resources, we collected $10 \mathrm{lf}, 31 \mathrm{lp}$, and $14 \mathrm{fh}$ mutants that were categorized phenotypically in the TOMATOMA mutant database. We then re-quantified the phenotypic characteristics of the mutants (see materials and methods). Five of the $l f$ mutants produced at least one more leaf on the primary shoot (PS) than Micro-Tom in both spring and autumn growth seasons (Figure 2a,b and Table S2). Twenty-two lp mutants revealed that plant weight was at least 1.5 times higher than that of the control plant (Figure $2 \mathrm{c}$ and Table 1). Seven $f h$ mutants produced abnormal floral organs, such as sepal-like petals and multiple inflorescence branches (Figure 2d, and Table 1).

a

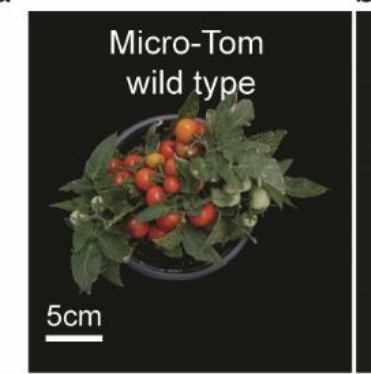

C

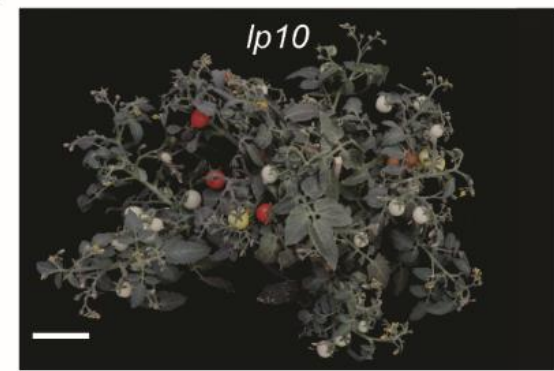

b

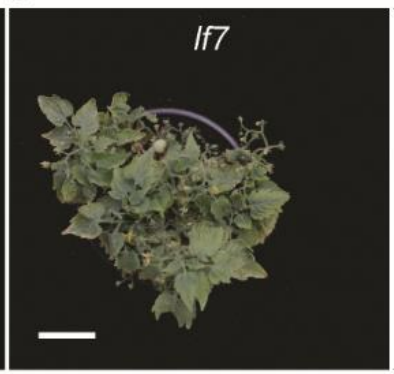

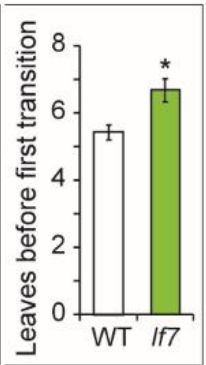

d
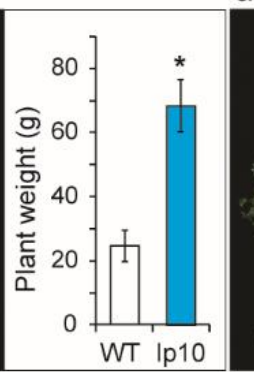

e
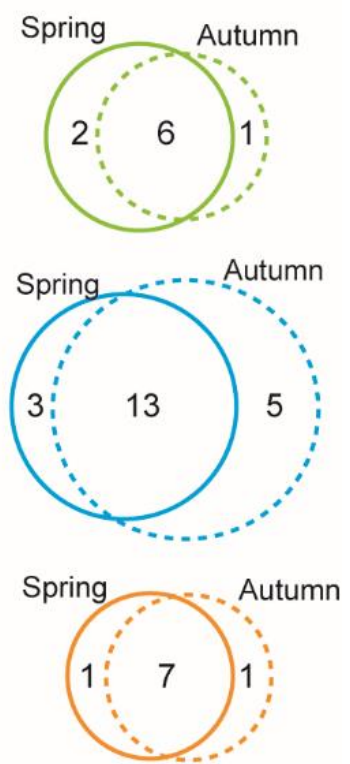

Figure 2. Flowering time variants showing $l f, l p$, and $f h$ mutants. (a-d) Representative plant growth showing Micro-Tom as wild type (a), late-flowering plant growth of $l f 7$ (b, left) with the comparative graph for leaves produced by the primary shoot meristem (PSM) with Micro-Tom (b, right), large plant growth of lp10 (c, left) with the comparative graph for plant weight with Micro-Tom (c, right), and floral homeotic phenotype of $f$ h 8 with the comparative image of inflorescences with Micro-Tom (d). (e) Venn diagrams showing the number of mutant lines producing more than 6 leaves by PSM in spring and autumn experiments. Colored solid-line circles indicate the spring experiment. Colored dashed-line circles indicate the autumn experiment. Three mutant groups categorized as If (green line circles), lp (blue line circles), and fh mutants (orange line circles) ${ }^{*}, p<0.05$. 
Table 1. Flowering time is associated with three phenotypic groups categorized with late-flowering, large plant, and floral homeotic phenotypes.

\begin{tabular}{ccccc}
\hline & $\begin{array}{c}\text { Number of } l f \\
\text { Mutants }\end{array}$ & $\begin{array}{c}\text { Number of } l p \\
\text { Mutants }\end{array}$ & $\begin{array}{c}\text { Number of } f h \\
\text { Mutants }\end{array}$ & Total \\
\hline Number of each phenotypic & 5 & 22 & 11 & 38 \\
$\begin{array}{c}\text { category } \\
\text { Late-flowering mutants }\end{array}$ & 5 & 13 & 7 & 25 \\
Percentage with late flowering & $100 \%$ & $59 \%$ & $64 \%$ & $66 \%$ \\
\hline
\end{tabular}

To examine whether their phenotypic categories were related to flowering time, the thirty-eight phenotypically re-examined mutants were used to quantify flowering time with the number of leaves on the PS in both spring and autumn growth seasons. Notably, Micro-Tom produced 4.9 and 5.1 leaves by PSM in spring and autumn. The five If mutants produced more than six leaves on the PS in both seasons. Thirteen (59\%) lp mutants and seven (64\%) fh mutants developed more than six leaves in PS in both seasons (Figure 2e and Table 1). Notably, three $l f$, eight $l p$, and two fh mutants flowered late in only one season (Figure 2e). These mutants may have been affected by environmental signals, such as seasonal cues. Because the plants were grown under greenhouse conditions in two seasons, we did not count single-season late-flowering mutants as late-flowering variants for further yield trials (Figure 2e). Therefore, the 25 mutants showing late-flowering, large plant, and floral homeotic phenotypes consistently prolonged the time point of change to the reproductive phase. These 25 mutants were used as genetic resources for screening high-yielding variants.

\subsection{Late-Flowering Continuum to Show the Trends of Biomass and Yield}

To study the relationship between the time of floral phase transition and bio-production, such as biomass and fruit yield, all the late-flowering variants were grown in the soil controlled by a drip irrigation system in the greenhouse [24]. The mutant genotypes were rearranged according to leaf production from PSM as a continuum of phase transition time to floral organs (Figure 3a). Based on this continuum, we tried to read the trends in plant weight, total yield, and Brix values. The overall trend of biomass yields among mutants, as measured by plant weight, showed gradual increments from weak to strong delayed flowering time (Figure 3b).

The trend of total yields among the late-flowering variants showed a bell-shaped curve, which means a gradual increase to the highest yield values from weaker to certain middle-late-flowering variants and then a gradual decrease in the values for the stronger late-flowering mutants (Figure 3c). The soluble sugar content, named Brix, showed no noticeable tendency on the late-flowering continuum (Figure $3 \mathrm{~d}$ ).

To determine the effects of yield on axillary shoot growth, we counted all axillary shoots produced on the axils of leaves in the main shoots. The axillary shoot numbers gradually increased in the more delayed-flowering-time mutants. All three phenotypic categories showed a uniformly linear increase in the axillary shoot numbers, which was a trend similar to the increase in leaf number (Figure 3e).

Because the optimal trends may change between environments, we performed a second set of yield trials with late-flowering variants in autumn under greenhouse conditions. The same pattern was observed between plant weight and total yield according to the floral transition time in PSM, even though plant weight and fruit harvest were relatively higher in controls than in the spring-summer season because less light intensity activated more vegetative growth in the autumn-winter season. Interestingly, lp5 had an additional highyield genotype with moderate late-flowering time (Supplementary Materials Figure S1). 
a

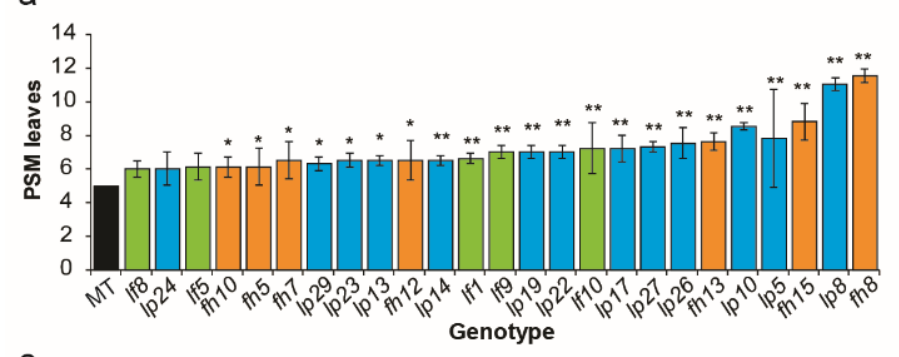

C

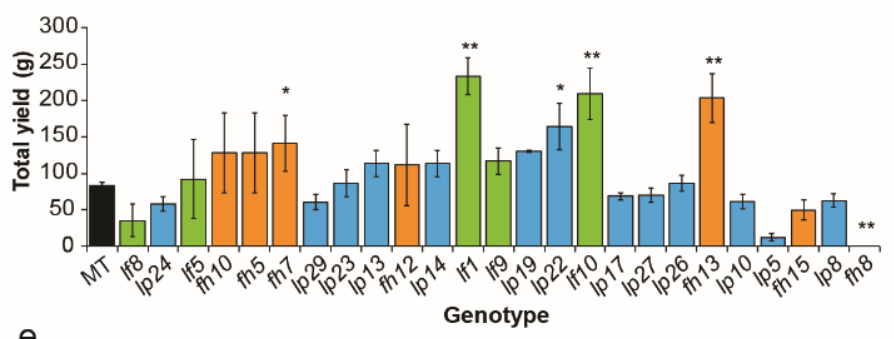

e

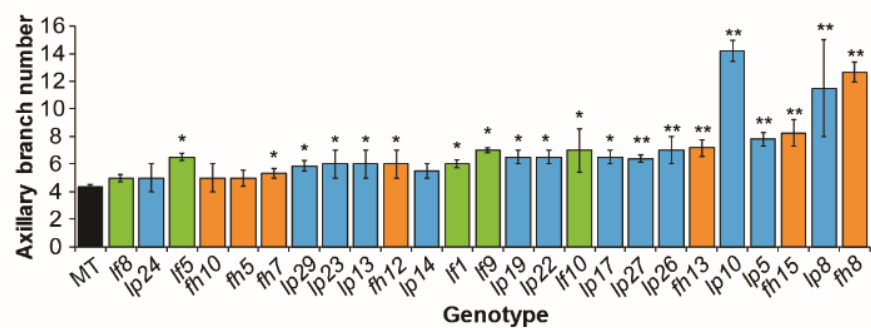

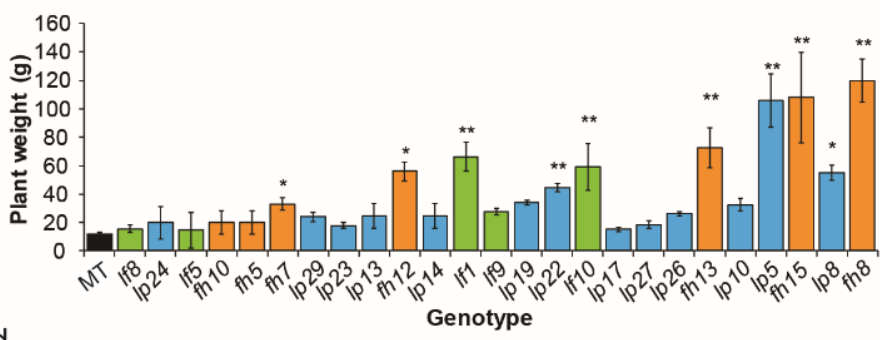

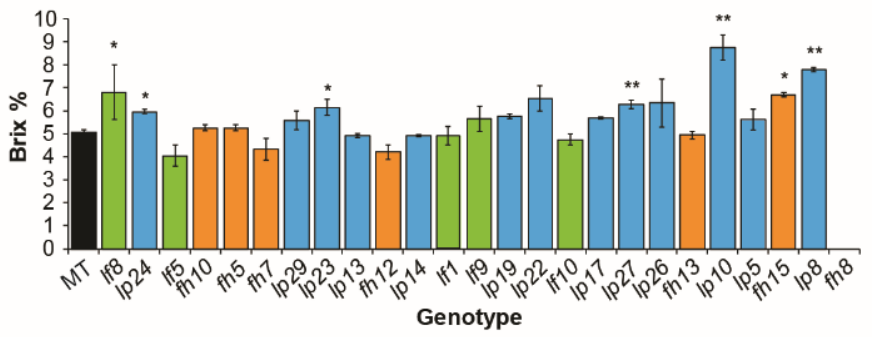

Figure 3. Quantifications of tomato yield-related traits of late-flowering variants in spring. Statistical comparisons of flowering time (a), plant weight (b), total yield (c), Brix value (d), axillary branch number (e) collected from late-flowering variants. Three phenotypic mutant groups categorized as lf (green color bars), fh (orange color bars), and lp mutants (blue color bars) were arranged according to leaf number produced by PSM. Mean values ( \pm s.e.m.) were compared to those for wild-type and mutants categorized into three groups using Student's $t$-tests $\left({ }^{*}, p<0.05 ; * *, p<0.01\right)$.

\subsection{Pattern of SFT and SP5G Expression Among Flowering Time Variants}

$S F T$ is the integrator of flowering signals cascading from endogenous and environmental conditions and antagonistically interacts with SP and SP5Gs $[25,26]$. The level of florigen activation affects the flowering times of PSM and SYM in determinate tomato and has been translated to the optimal curve of tomato yield according to the time of flowering [1]. To estimate SFT and SP5Gs expression in the flowering time variants, mature green leaves were sampled and the amount of SFT transcripts was quantified.

Most of the late-flowering variants displayed downregulated SFT expression. Ten genotypes showed lower SFT expression, but four genotypes showed higher expression than the wild type. The two lf mutants transcribed normal amounts of SFT under lateflowering conditions (Figure 4a). Interestingly, none of the variants showed upregulated SP5G expression in mature leaves (Figure 4b). Notably, SP5G2 and SP5G3 transcripts were weakly detected in all variants' mature leaves except SP5G2 expression of fh13 and SP5G3 expression of lp10 (Figure S2). 
a

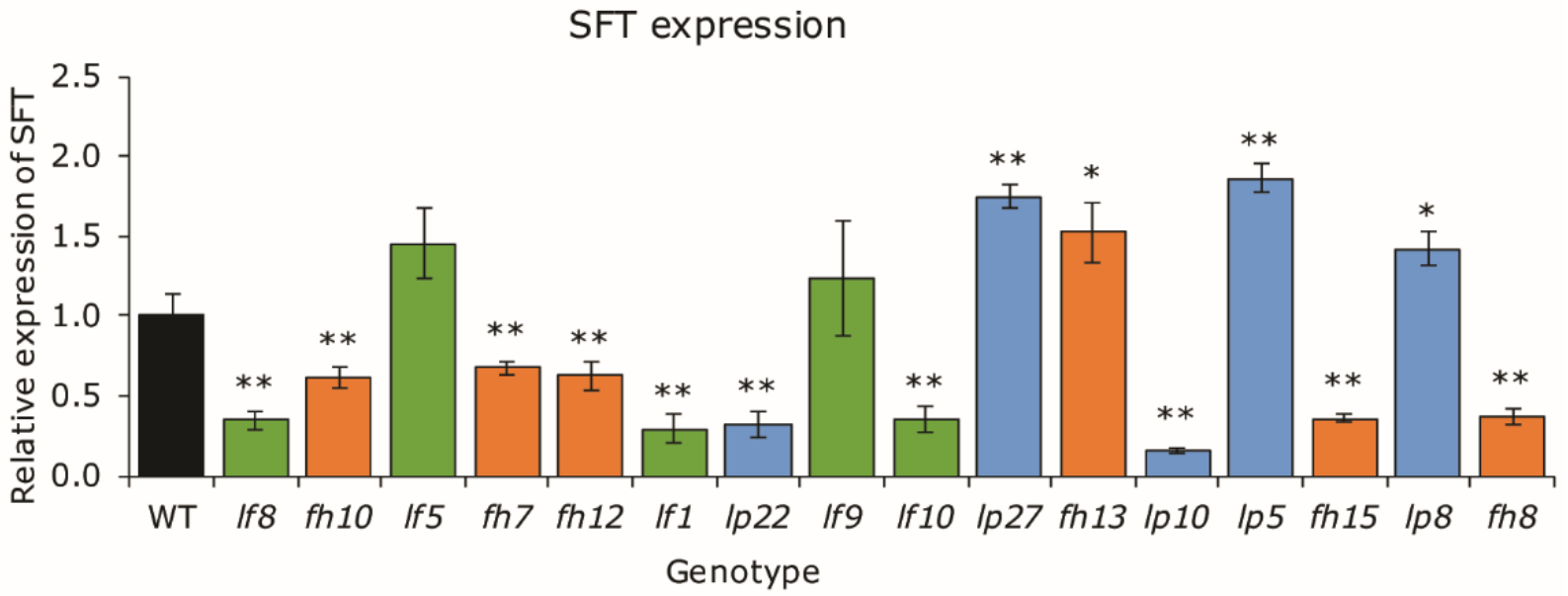

b

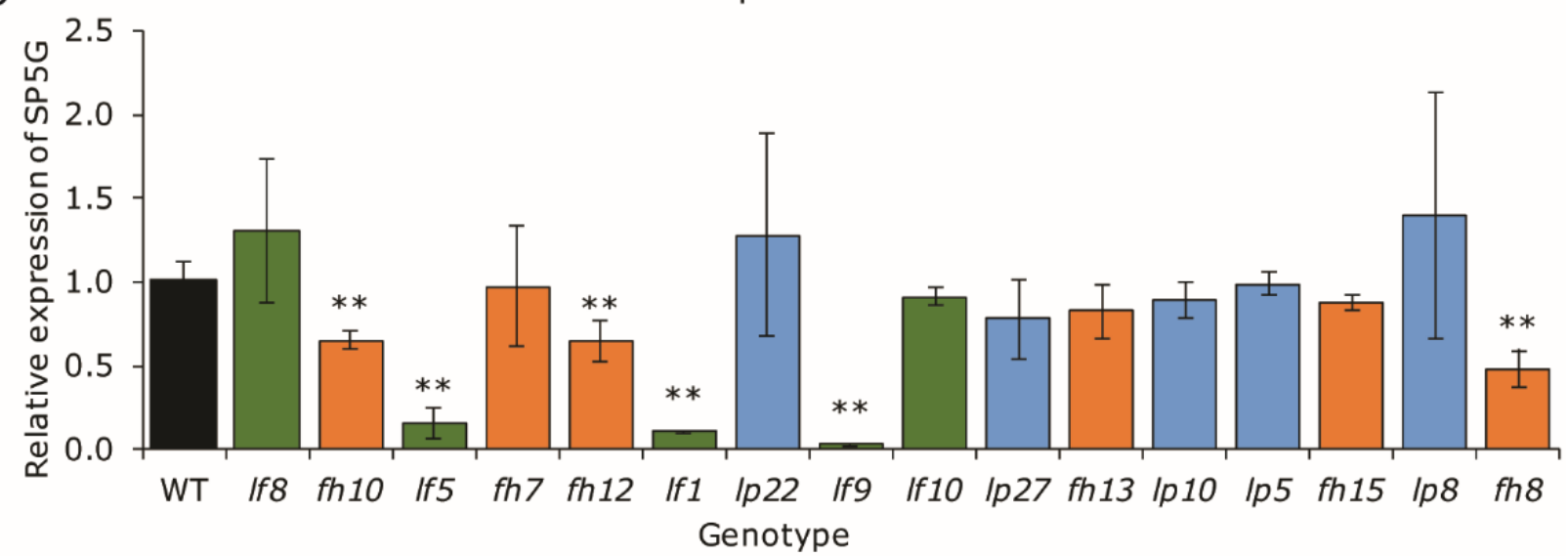

Micro-Tom (Wild Type)

If mutants

fh mutants

Ip mutants

Figure 4. Examination of SFT (a) and SP5G (b) expression from mature leaves to assess the correlation between flowering time and floral hormone. Three phenotypic mutant groups categorized as $l f$ (green color bars), fh (orange color bars), and $l p$ mutants (blue color bars) were arranged by PSM according to leaf number produced. Mean values $( \pm \mathrm{SD})$ were compared to those for wild-type and mutants categorized into three groups using Student's $t$-tests $\left({ }^{*}, p<0.05 ;{ }^{* *}, p<0.01\right)$.

\subsection{Isolation of Tomato Yield Optimum Among lp and fh Mutants}

Interestingly, the collected $l p$ and $f h$ mutants had positive effects on bio-yield within the time period of transitioning to the floral phase in Micro-Tom. To clarify whether the yield optimum of the two groups of mutants resulted from late-flowering time or other clues, we conducted a third experiment with the mutants under the ideal growth conditions in the greenhouse. The flowering time continuum of the mutants translated to similar trends in total yields and plant weight compared to the previous two experiments (Figure 5a-f). Notably, Brix content clearly increased according to late-flowering time continuums in the third trial (Figure 5e).

To examine whether biomass and fruit yields were correlated with axillary shoot or flower production, the trends of flowering time and plant weight were compared with the late-flowering continuum using $l p$ and $f h$ mutants. The $l p$ mutant trend in plant weight was not only associated with flowering time but also showed a similar trend of total axillary shoot number showing the production of axillary shoots and sympodial shoots among mutants (Figure $5 \mathrm{~b}, \mathrm{f})$. Interestingly, $l p 22, l p 10$, and $l p 8$ produced relatively higher numbers of axillary shoots and plant weight than other late-flowering variants on the main shoot (Figure 5f). 
a

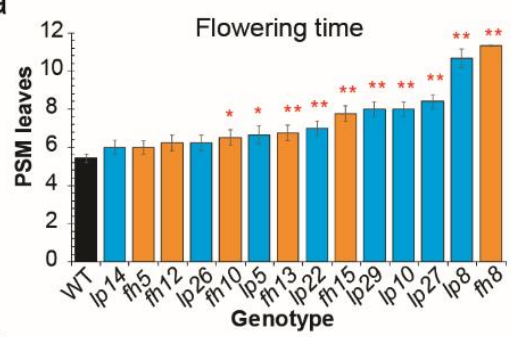

d

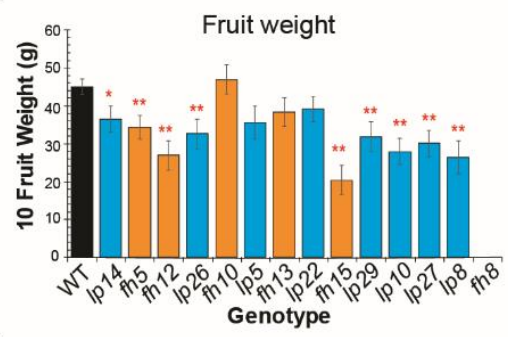

b

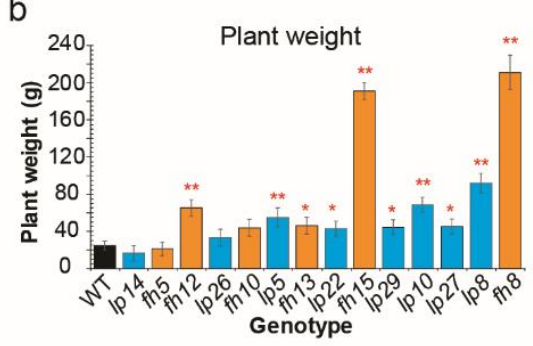

e

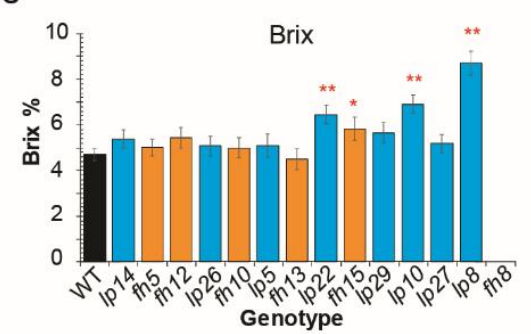

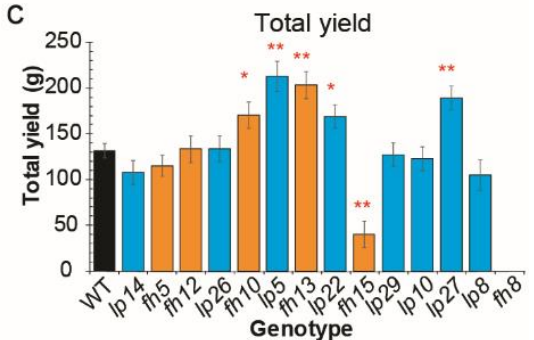

f

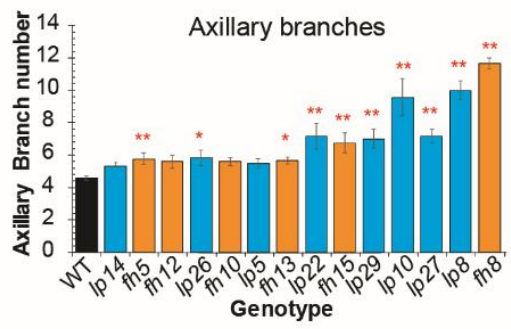

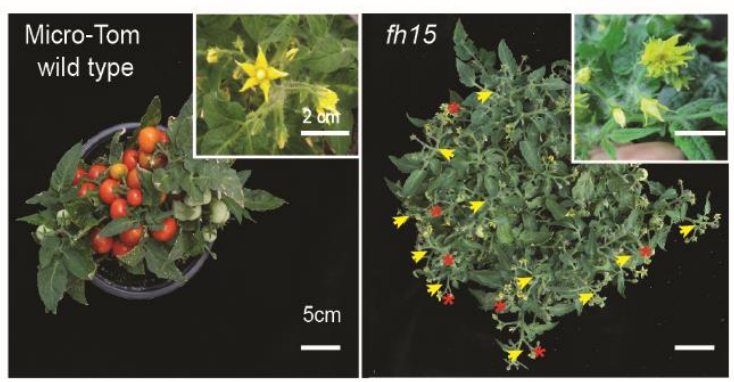

Micro-Tom (Wild Type)

Ip mutants

fh mutants

Figure 5. Exploring yield dynamics determining the relationship with the late-flowering continuum with $l p$ and $f h$ mutants. Statistical comparison of leaf numbers $( \pm$ s.e.m) indicating the flowering time of mutants and arrangement in ascending order of flowering time (a). (b-f) Statistical comparisons of the value of plant weight (b), total yield (c), fruit weight (d), Brix value (e), and axillary branch number (f). Representative images of Micro-tom shoots and flowers (left) and fh15 plant with fasciated flower and increased inflorescence indicating the higher branching was not compensated by the flowering time. Fasciated flowers and inflorescence were magnified in white line box (g). Yellow arrowheads indicate branches on the inflorescence. Red asterisks indicate fasciated flowers. Mean values ( \pm s.e.m.) were compared to those for wild-type and late-flowering mutants using Student's $t$-tests $\left({ }^{*} p<0.05,{ }^{* *} p<0.01\right)$. Multiple-comparison analyses were also conducted using Tukey-Kramer test $(p<0.05$; Table S3).

The $f h$ mutants also showed more axillary shoots according to the delay in flowering time (Figure 5a,f). Moreover, they showed relatively higher plant weights than other $l p$ mutants, producing a similar number of leaves in the primary shoot (Figure 5b). For example, fh15 weighed much higher than $l p 29$ and $l p 10$, even though $f h 15$ produced slightly fewer leaves in the primary shoot and fewer axillary shoots on the main shoot than $1 p 29$ and lp10. The mutants $f h 8$ and $f h 15$ developed more inflorescence branches and floral organs because their reproductive organs were continuously produced after floral transitions, resulting in extremely high plant weights (Figures $2 d$ and $5 b, g$ ).

Using statistical analysis, we were able to isolate significantly higher yields in $\operatorname{lp} 5$, and $l p 22$ among the $l p$ mutants, which produced more leaves from PSM and more axillary shoots on whole plants than the control, but fewer leaves and axillary shoots than the extremely large plants such as $\operatorname{lp} 8$ and $\operatorname{lp} 10$ (Figures $5 \mathrm{f}$ and $6 \mathrm{a}$ ). The mutants $f h 10$ and fh13 also produced significantly higher tomato yields compared to the control, with minor biomass increments showing more leaf and axillary shoots from minor delayed flowering (Figure $5 c, f)$. The mutants $f h 8$ and $f h 15$ developed extra floral organs that produced sepallike floral organs, resulting in significantly high biomass production but not high yields due to unbalanced fruit production and organ development (Figures $2 \mathrm{~d}$ and $5 \mathrm{~g}$ ). 
a

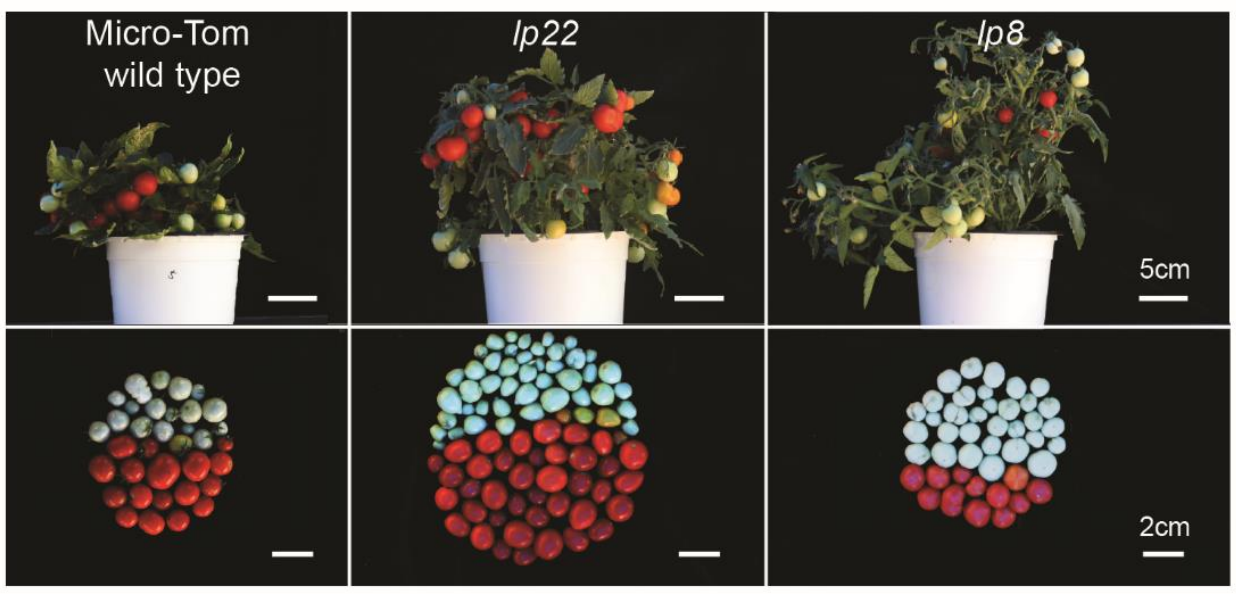

b

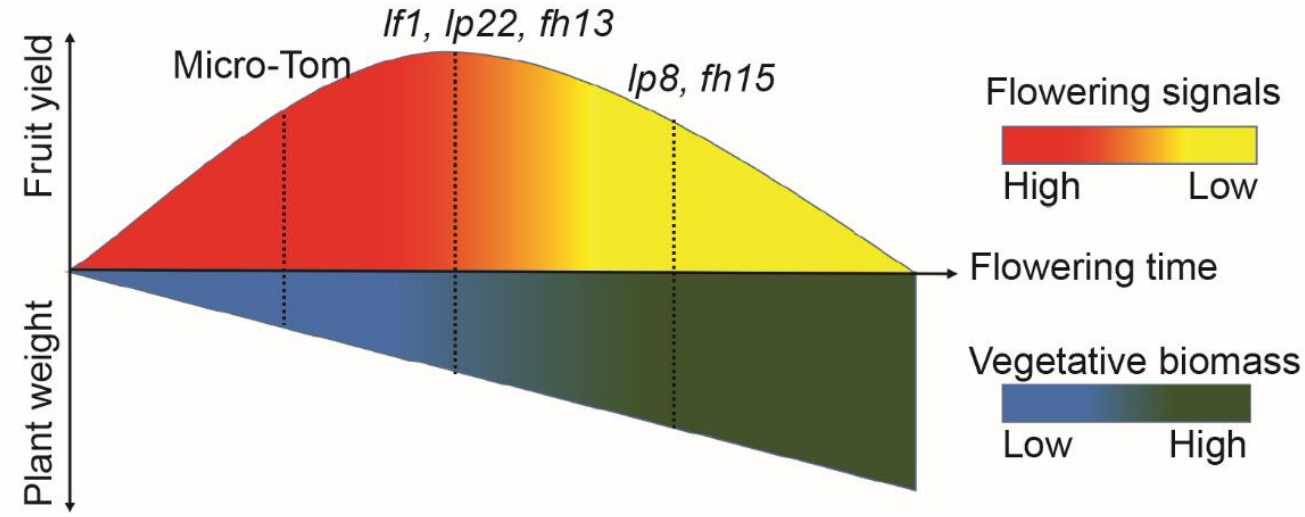

Figure 6. Representative tomato yields and yield dynamic model with flowering time variants. (a) Representative vegetative biomass (top images) and tomato yield (bottom images) with three different flowering time variants. Notably, lp22 shows a balanced growth with optimal late-flowering, maximum yields, and optimal-increment of biomass (middle images). $1 p 8$ was grown with extremely late-flowering, lower yield, and higher amount of biomass (right images). Upper size bar, $5 \mathrm{~cm}$; bottom size bar, $2 \mathrm{~cm}$. (b) Yield dynamics model with plant performance under varying flowering time (Figure S3, Table S4). This model suggests the optimal flowering time variants such as $l f 1, l p 22$, and fh13 get the optimal vegetative mass and fruit sets (dashed lines) bearing well-balanced vegetative and reproductive organ production for a higher yield. This model also suggests that an extremely late-flowering time, such as that of $l p 8$ and $f h 15$ plants, produces excessive vegetative mass but achieves less fruit yields.

\subsection{Correlation between Flowering Time and Tomato Yields}

To examine whether the yield-related traits' values were statistically associated with flowering time delay, we evaluated the correlation between flowering time and each trait out of five yield-related traits using data collected from three seasons. The bivariate correlation assay indicated that plant weight and Brix were positively correlated, but total yield and fruit weight were negatively correlated with a flowering time delay (Table S4, Figure S3). Moreover, the non-linear regression analysis indicated that the association between flowering time and total yield showed a statistical curve change determining the fitness of total yield on the flowering time variations (Figure S3).

Altogether, we suggest an expanded "yield dynamic model" with flowering time variants. The model suggested that prolonged flowering time positively increased biomass yields, such as vegetative and reproductive organ production, but uncoupled tomato fruit yields in mutants with a later flowering time than the maximum yield time. The model also suggested that tomato yield dynamics could be caused by flowering time variants, indicating that universal or novel flowering signals could affect yield increments in the mutants of flowering time regulators (Figure 6b). 


\section{Discussion}

We isolated 25 delayed flowering variants from $5 \mathrm{lf}, 22 \mathrm{lp}$, and $11 \mathrm{fh}$ mutants. Of these, 59\% lp mutants and $64 \%$ fh mutants were highly associated with regulating the transition time to the floral phase, and they were associated with an increase in biomass. Universally, flowering plants produced more biomass in late-flowering variants because prolonged vegetative development gave the plant more time to produce leaves and axillary shoots, thereby increasing plant size. Solanaceae species, such as tomato, could exhibit increased biomass in late-flowering mutants because the species undergoes multiple floral transitions in sympodial and axillary shoot growth throughout their lifespan after the first floral transition in PSM [27]. Specifically, $l p 8$ and $l p 10$ developed relatively more leaves and axillary shoots, resulting in high biomass (Figure $3 \mathrm{~b}, \mathrm{e}$ ). The mutants $l p 8$ and $l p 10$ produced a large number of axillary shoots under late-flowering time, indicating that they could exhibit axillary shoot dormancy under prolonged flowering conditions (Figures $3 \mathrm{e}$ and $5 \mathrm{f}$ ).

Our new $f h$ mutants not only showed greater production of inflorescences and floral organs but also produced more leaves and axillary shoots on the main shoots (Figure 5). Mutations in falsiflora and uniflora led to the development of many leaves in PSM and failed to produce normal flowers and inflorescences [28,29]. The mutation resulted in multiple inflorescence branches, and macrocalyx led to the production of a large sepal and delayed minor floral transition time in tomato [30,31]. The mutants fh12 and fh15 developed additional floral or reproductive organs, and $f h 8$ endlessly yielded leaf-like floral organs and inflorescence branches after the floral transition from vegetative shoots (Figures $2 \mathrm{~d}$ and $5 \mathrm{~b}$ ). The increased production of vegetative organs during development caused increased biomass in $f h$ mutants than in the other late-flowering mutants. This effect is similar to the typical floral defective mutant 'falsiflora' and 'anantha', which show several additional leaf-like floral organs and inflorescence branches after the late floral transition from vegetative meristems [30,32,33].

However, the higher biomass of both vegetative organs and a high number of reproductive organs did not directly contribute to tomato fruit yields because of the loss in the balance between organ production and fruit setting. Our flowering time continuums using 25 late-flowering variants isolated lf1, lf10, lp2, lp5, lp22, and fh13 as high-yielding genotypes (Figures $3 \mathrm{c}$ and $5 \mathrm{c}$ ). All six mutants flowered at similar flowering time regimes and produced almost the same axillary shoots in whole plants, resulting in relatively high yields, unlike the other extremely late-flowering mutants or the control (Figure 3c,e and Figure $5 \mathrm{c}, \mathrm{f})$. These results indicate that the $l f, l p$, and $f h$ mutants can be optimized for the development time of leaf and axillary shoot production, translating into high tomato yield with optimal transition time to flower because flowering time is highly associated with leaf and axillary shoot production.

Our late-flowering variants could be rearranged with a continuum of flowering time to analyze the pattern of biomass and fruit yield, and they could be used to identify mutants with high yielding biomass and fruit harvest as genetic resources for future breeding. Lateflowering mutants produced approximately 6-11 leaves in the primary shoot, compared with five leaves of the wild type. This continuum could be translated into a trend of yields, such as fruits and biomass. Interestingly, the highest and optimal harvests were selected on the top of the bell-shaped curve of total yield among the arranged mutants according to flowering time, which we summarized into a new yield model (Figure 6b). Our yield dynamics from flowering time variants showed a trend similar to that of the dosage effects of tomato florigen activation and antagonism [1].

Molecularly, the flowering time coupled with plant size and floral organ production could not be only attained using flowering signals, such as florigen activation signals, but might also gain other flowering signals from floral identity genes and phase transitioning genes [32]. Our molecular evidence from florigen and antiflorigen expression also shows that the SFT and SP5G expression varied among all late-flowering-low yielding and optimal flowering-high yielding mutants, indicating that these mutants are comprised of malfunctions in mainly the florigen signal pathway or other parallel pathway regulation 
flowering. This indicated that our new dynamics modeled an additional scope from florigen dosage models because optimum yields could be achieved by balancing floral phase change time by regulating florigen signals and other flowering signals (Figure 6) [1].

\section{Conclusions}

Our late-flowering variants rearranged with a continuum of flowering time indicated scope for genetic mutants' isolation with high biomass and fruit harvest. We suggested a "yield dynamic model" representing the optimal balance between the vegetative and reproductive phases determining tomato productivity. This balanced yield model could be expanded with a vast potential to fine-tune the optimum flowering time for higher yields of the Solanaceae family, including tomato and eggplant. The mutation of flowering time variants can be identified through mapping using novel sequencing tools. Flowering or floral regulator genes can then be edited to induce late-flowering to achieve beneficial mutations using the CRISPR/Cas9 system, which is short for clustered regularly interspaced short palindromic repeats and CRISPR-associated protein 9. Ultimately, our models are applicable to all flowering crops in which floral transition is controlled by genetic regulation. Further genetic and molecular studies on flowering time variants will provide fundamental genetic cues for fine-tuning and optimizing yields in tomato and other crops, and they will provide evidence of the power of our balanced model for future crop breeding.

Supplementary Materials: The following are available online at https:/ / www.mdpi.com/2073-439 5/11/2/285/s1, Figure S1. Quantifications of yield-related traits from late-flowering variants in the autumn season. Statistical comparisons of flowering time (a), total yield (b), plant weight (c), Brix value (d) collected from late-flowering variants. Three phenotypic mutant groups categorized as $l f$ (green color bars), fh (orange color bars), and lp mutants (blue color bars) were arranged by PSM according to leaf number produced. Mean values ( \pm s.e.m.) were compared to those for wild-type and mutants categorized into three groups using Student's t-tests $\left({ }^{*}, p<0.05 ;{ }^{* *}, p<0.01\right)$. Figure S2. Semi-quantitative RT-PCR analysis of SP5G2 and SP5G3 expression levels in mature leaves Genotypes arranged according to leaf production in PSM. Two biological replicates (1st and 2nd) were used for semi-qRT-PCR. Figure S3. Statistical modeling of total yield by flowering time. (a) Linear and quadratic projection of plant weight using three-season experiments. (b) quadratic projection of plant weight using three-season experiments. Table S1. Primer information for the qRT-PCR experiments. Table S2. Late-flowering mutants isolated from three mutant groups with different morphology received from TOMATOMA. Table S3. Statistical grouping of flowering time, total yield, plant weight, 10 fruit weight, and Brix value (Grouping was done using Tukey-Kramer HSD at a confidence interval of 0.05). Table S4. Bivariate correlation analysis between flowering time and four traits in all seasons.

Author Contributions: S.R., J.H., and S.J.P. designed the research and performed the experiments; Y.K.L., S.K.O., Y.J.K., D.H.K., and K.K. contributed to the phenotyping and tomato yield trial; C.M.K. and J.H.B. performed the systemic control for tomato growth; S.R. and S.J.P. wrote the manuscript, with editing contributions from all authors. All authors have read and agreed to the published version of the manuscript.

Funding: This research was supported by a grant from the Next-Generation BioGreen 21 Program (PMBC, grant no. PJ0133032020; SSAC, Grant No. PJ0134212020) funded by the Rural Development Administration to S.J.P. and J.H.B., and the 'National Research Foundation of Korea (grant no. NRF-2016R1C1B2015877) funded by the Ministry of Science, ICT and Future Planning' to S.J.P. and the 'National Research Foundation of Korea (grant no. NRF-2017R1A4A1015594, NRF2019M3E5D5067214) funded by the Korean Ministry of Science, ICT, and Future Planning' to D.H.K. and K-S.K.

Institutional Review Board Statement: Not applicable.

Informed Consent Statement: Not applicable.

Acknowledgments: The authors would like to thank the members of Park's Lab for plant care. We also thank Ken Hoshikawa and TOMATOMA for providing all the Micro-Tom mutant resources used in this study. 
Conflicts of Interest: The authors have no conflicts of interest to declare.

\section{References}

1. Park, S.J.; Jiang, K.; Tal, L.; Yichie, Y.; Gar, O.; Zamir, D.; Eshed, Y.; Lippman, Z.B. Optimization of crop productivity in tomato using induced mutations in the florigen pathway. Nat. Genet. 2014, 46, 1337-1342. [CrossRef]

2. Huijser, P.; Klein, J.; Lönnig, W.E.; Meijer, H.; Saedler, H.; Sommer, H. Bracteomania, an inflorescence anomaly, is caused by the loss of function of the MADS-box gene squamosa in Antirrhinum majus. EMBO J. 1992, 11, 1239-1249. [CrossRef]

3. Shrestha, R.; Gómez-Ariza, J.; Brambilla, V.; Fornara, F. Molecular control of seasonal flowering in rice, arabidopsis and temperate cereals. Ann. Bot. 2014, 114, 1445-1458. [CrossRef] [PubMed]

4. Salehi, H.; Ransom, C.B.; Oraby, H.F.; Seddighi, Z.; Sticklen, M.B. Delay in flowering and increase in biomass of transgenic tobacco expressing the Arabidopsis floral repressor gene Flowering Locus C. J. Plant Physiol. 2005, 162, 711-717. [CrossRef] [PubMed]

5. Xue, W.; Xing, Y.; Weng, X.; Zhao, Y.; Tang, W.; Wang, L.; Zhou, H.; Yu, S.; Xu, C.; Li, X.; et al. Natural variation in Ghd7 is an important regulator of heading date and yield potential in rice. Nat. Genet. 2008, 40, 761-767. [CrossRef]

6. Ye, J.; Niu, X.; Yang, Y.; Wang, S.; Xu, Q.; Yuan, X.; Yu, H.; Wang, Y.; Wang, S.; Feng, Y. Divergent Hd1, Ghd7, and DTH7 alleles control heading date and yield potential of Japonica rice in northeast China. Front. Plant Sci. 2018, 9, 35. [CrossRef] [PubMed]

7. Yano, M.; Katayose, Y.; Ashikari, M.; Yamanouchi, U.; Monna, L.; Fuse, T.; Baba, T.; Yamamoto, K.; Umehara, Y.; Nagamura, Y.; et al. Hd1, a major photoperiod sensitivity quantitative trait locus in rice, is closely related to the Arabidopsis flowering time gene CONSTANS. Plant Cell 2000, 12, 2473-2483.

8. Koo, B.H.; Yoo, S.C.; Park, J.W.; Kwon, C.T.; Lee, B.D.; An, G.; Zhang, Z.; Li, J.; Li, Z.; Paek, N.C. Natural variation in OsPRR37 regulates heading date and contributes to rice cultivation at a wide range of latitudes. Mol. Plant 2013, 6, 1877-1888. [CrossRef]

9. Gao, H.; Jin, M.; Zheng, X.-M.; Chen, J.; Yuan, D.; Xin, Y.; Wang, M.; Huang, D.; Zhang, Z.; Zhou, K.; et al. Days to heading 7, a major quantitative locus determining photoperiod sensitivity and regional adaptation in rice. Proc. Natl. Acad. Sci. USA 2014, 111, 16337-16342. [CrossRef]

10. Melzer, S.; Lens, F.; Gennen, J.; Vanneste, S.; Rohde, A.; Beeckman, T. Flowering-time genes modulate meristem determinacy and growth form in Arabidopsis thaliana. Nat. Genet. 2008, 40, 1489-1492. [CrossRef]

11. Soyk, S.; Müller, N.A.; Park, S.J.; Schmalenbach, I.; Jiang, K.; Hayama, R.; Zhang, L.; Van Eck, J.; Jiménez-Gómez, J.M.; Lippman, Z.B. Variation in the flowering gene SELF PRUNING 5G promotes day-neutrality and early yield in tomato. Nat. Genet. 2017, 49, 162-168. [CrossRef] [PubMed]

12. Prusinkiewicz, P.; Erasmus, Y.; Lane, B.; Harder, L.D.; Coen, E. Evolution and development of inflorescence architectures. Science 2007, 316, 1452-1456. [CrossRef] [PubMed]

13. Yeager, A.F. Determinate growth in the tomato. J. Hered 1927, 18, 263-265. [CrossRef]

14. Thouet, J.; Quinet, M.; Ormenese, S.; Kinet, J.M.; Périlleux, C. Revisiting the involvement of Self-pruning in the sympodial growth of tomato. Plant Physiol. 2008, 148, 61-64. [CrossRef]

15. Krieger, U.; Lippman, Z.B.; Zamir, D. The flowering gene SINGLE FLOWER TRUSS drives heterosis for yield in tomato. Nat. Genet. 2010, 42, 459-463. [CrossRef] [PubMed]

16. Taoka, K.I.; Ohki, I.; Tsuji, H.; Furuita, K.; Hayashi, K.; Yanase, T.; Yamaguchi, M.; Nakashima, C.; Purwestri, Y.A.; Tamaki, S.; et al. 14-3-3 proteins act as intracellular receptors for rice Hd3a florigen. Nature 2011, 476, 332-335. [CrossRef] [PubMed]

17. Cao, K.; Yan, F.; Xu, D.; Ai, K.; Yu, J.; Bao, E.; Zou, Z. Phytochrome B1-dependent control of SP5G transcription is the basis of the night break and red to far-red light ratio effects in tomato flowering. BMC Plant Biol. 2018, 18, 158. [CrossRef] [PubMed]

18. Lemmon, Z.H.; Park, S.J.; Jiang, K.; Van Eck, J.; Schatz, M.C.; Lippman, Z.B. The evolution of inflorescence diversity in the nightshades and heterochrony during meristem maturation. Genome Res. 2016, 26, 1676-1686. [CrossRef] [PubMed]

19. Saito, T.; Ariizumi, T.; Okabe, Y.; Asamizu, E.; Hiwasa-Tanase, K.; Fukuda, N.; Mizoguchi, T.; Yamazaki, Y.; Aoki, K.; Ezura, H. TOMATOMA: A Novel Tomato Mutant Database Distributing Micro-Tom Mutant Collections. Plant Cell Physiol. 2011, 52, 283-296. [CrossRef]

20. Shikata, M.; Ezura, H. Micro-tom tomato as an alternative plant model system: Mutant collection and efficient transformation. In Methods in Molecular Biology; Humana Press Inc.: Totowa, NY, USA, 2016; Volume 1363, pp. 47-55.

21. Menda, N.; Semel, Y.; Peled, D.; Eshed, Y.; Zamir, D. In silico screening of a saturated mutation library of tomato. Plant J. 2004, 38, 861-872. [CrossRef] [PubMed]

22. Shinozaki, Y.; Hao, S.; Kojima, M.; Sakakibara, H.; Ozeki-Iida, Y.; Zheng, Y.; Fei, Z.; Zhong, S.; Giovannoni, J.J.; Rose, J.K.; et al. Ethylene suppresses tomato (Solanum lycopersicum) fruit set through modification of gibberellin metabolism. Plant J. 2015, 83, 237-251. [CrossRef]

23. Meissner, R.; Jacobson, Y.; Melamed, S.; Levyatuv, S.; Shalev, G.; Ashri, A.; Elkind, Y.; Levy, A. A new model system for tomato genetics. Plant J. 1997, 12, 1465-1472. [CrossRef]

24. Rothan, C.; Just, D.; Fernandez, L.; Atienza, I.; Ballias, P.; Lemaire-Chamley, M. Culture of the Tomato Micro-Tom Cultivar in Greenhouse. Plant Signal Transduct. 2016, 1363, 7-10.

25. Lifschitz, E.; Eviatar, T.; Rozman, A.; Shalit, A.; Goldshmidt, A.; Amsellem, Z.; Alvarez, J.P.; Eshed, Y. The tomato FT ortholog triggers systemic signals that regulate growth and flowering and substitute for diverse environmental stimuli. Proc. Natl. Acad. Sci. USA 2006, 103, 6398-6403. [CrossRef] 
26. Cao, K.; Cui, L.; Zhou, X.; Ye, L.; Zou, Z.; Deng, S. Four tomato FLOWERING LOCUS T-like proteins act Antagonistically to regulate floral initiation. Front. Plant Sci. 2016, 6, 1213. [CrossRef] [PubMed]

27. Xu, C.; Park, S.J.; Van Eck, J.; Lippman, Z.B. Control of inflorescence architecture in tomato by BTB/POZ transcriptional regulators. Genes Dev. 2016, 30, 2048-2061. [CrossRef] [PubMed]

28. Liljegren, S.J.; Gustafson-Brown, C.; Pinyopich, A.; Ditta, G.S.; Yanofsky, M.F. Interactions among APETALA1, LEAFY, and TERMINAL FLOWER1 specify meristem fate. Plant Cell 1999, 11, 1007-1018. [PubMed]

29. Quinet, M.; Kinet, J.M.; Lutts, S. Flowering response of the uniflora:blind:self-pruning and jointless:uniflora:self-pruning tomato (Solanum lycopersicum) triple mutants. Physiol. Plant. 2011, 141, 166-176. [CrossRef] [PubMed]

30. Lippman, Z.B.; Cohen, O.; Alvarez, J.P.; Abu-Abied, M.; Pekker, I.; Paran, I.; Eshed, Y.; Zamir, D. The making of a compound inflorescence in tomato and related nightshades. PLoS Biol. 2008, 6, 2424-2435. [CrossRef]

31. Vrebalov, J.; Ruezinsky, D.; Padmanabhan, V.; White, R.; Medrano, D.; Drake, R.; Schuch, W.; Giovannoni, J. A MADS-box gene necessary for fruit ripening at the tomato ripening-inhibitor (rin) locus. Science 2002, 296, 343-346. [CrossRef] [PubMed]

32. Molinero-rosales, N.; Jamilena, M.; Zurita, S.; Capel, J.; Lozano, R. FALSIFLORA, the tomato orthologue of FLORICAULA and LEAFY, controls flowering time and floral meristem identity. Plant J. 1999, 20, 685-693. [CrossRef] [PubMed]

33. Park, S.J.; Jiang, K.K.; Schatz, M.C.; Lippman, Z.B. Rate of meristem maturation determines inflorescence architecture in tomato. Proc. Natl. Acad. Sci. USA 2012, 109, 639-644. [CrossRef] [PubMed] 